\title{
Tun und Nichttun bei Grillparzer. Eine Problematik des Biedermeier
}

\section{Wolfgang Nehring}

Welche geschichtsträchtige Epoche hat Grillparzer durchlebt! Bereits anderthalb Jahre nach dem Sturm auf die Bastille und dem Beginn der französischen Revolution hat er in die aufgeregte Welt geblickt, und vor seinem Lebensende hat der pensionierte Hofrat, der greise Weise von Wien, noch den deutsch-französischen Krieg und die Gründung des Deutschen Reiches wahrgenommen - ein Ereignis, das, nebenbei gesagt, auch einen Schlußstrich unter seine lebenslange österreichisch-deutsche Identitätssnot setzte. - Die Revolution von 1789 und ihre brutalen Folgen haben seine Kindheit beschäftigt. Der Schüler und junge Student hat die Niederlegung der römisch-deutschen Kaiserwürde und die Begründung des österreichischen Kaisertums durch Kaiser Franz II., nunmehr verjüngt zu Franz I. von Österreich, sowie die zweimalige Besetzung Wiens durch französische Armeen miterlebt. Während des Wiener Kongresses beschäftigte er sich bereits mit seiner unheimlichen Ahnfrau, die ihm die magischen Türen zum Dichterruhm öffnen, ihn aber auch gespenstisch durch sein Poetendasein verfolgen sollte. Zur Zeit der Revolution von 1848 schließlich ist nicht nur sein dichterischer Ehrgeiz, sondern auch sein poetischer Wille weitgehend erschöpft. Er registriert die politischen und sozialen Veränderungen, nimmt an der österreichisch-preußischen Auseinandersetzung und den Nationalitätenkonflikten innerhalb der Monarchie einigen Anteil, läßt sich die Huldigungen als "Staatsdichter", Orden und Ehrendoktorwürden gefallen, meldet sich aber nur noch selten zu Wort.

Der Dichter Grillparzer gehört daher fast ganz in die Restaurationszeit oder, wie man trotz gelegentlich angemeldeter Skrupel ${ }^{1}$ meines Erachtens bedenkenlos

1. Eine Übersicht über die frühe Biedermeier-Forschung und über die Bedenken, Grillparzer dem Biedermeier zuzuordnen, findet sich bei Ulrich Fülleborn, Das dramatische Geschehen im Werk Franz Grillparzers. Ein Beitrag zur Epochenbestimmung der deutschen Dichtung im 19. Jahrhundert. München 1966. - Walter Weiss ist auf unserem Symposium unter Hinweis auf Grillparzers Josefinismus erneut der Kategorisierung Grillparzers als Biedermeier-Autors entgegengetreten. Aber ohne die josefinische Tradition von Grillparzers Denken in Abrede zu stellen, ohne auch die klassische oder klassizistische Tendenz bei Grillparzer verkleinern zu wollen, - ohne also behaupten zu wollen, daß Grillparzer ganz im Biedermeier aufgeht, scheint mir der Begriff doch den vorherrschenden Geist seiner Dichtung plausibel zu beschreiben. Der von Weiss in der Nachfolge Herbert Seidlers propagierte Name "Österreichischer Vormärz" scheint mir für die lange Metternich-Zeit doch zu wenig charakteristisch. Vgl. zu dem ganzen 
sagen darf: ins literarische Biedermeier. ${ }^{2}$ Wenn der Begriff Biedermeier überhaupt einen Sinn hat, dann doch wohl bei Grillparzer, dem Wiener Dichter, der unter dem Druck der Metternichschen Restaurationspolitik leidend, durch Schikanen der Zensoren und des Hofs bis hinauf zu seiner Majestät dem Kaiser behindert, dennoch die loyalsten Dichtungen verfaßt hat, die sich ein Habsburger nur erträumen konnte oder hätte erträumen können, wenn seine Phantasie dafür ausgereicht hätte. Wer hätte wie Grillparzer die Selbsterhebung des Individuums abgestraft und das selbstgenügsame, natürliche, harmonische, von Zufriedenheit und Dankbarkeit bestimmte Leben propagiert? Wer hätte wie dieser eigentlich doch so gequälte und mürrische Dichter ein Österreich-Bild entworfen, das einem wohlgepflegten Blumengarten gleicht? ${ }^{3}$

Nun ist freilich von vornherein dem Mißverständnis vorzubeugen, als sei Grillparzer nicht nur ein Biedermeier-Dichter, sondern zugleich ein kleiner Biedermann gewesen, der vor den Brandstiftern seiner Zeit die Augen verschließt, ein Spießbürger und Drückeberger, der nicht denken kann und will. Es ist ausreichend bekannt, daß der Dichter, in josefinischer Tradition verwurzelt, sich selbst am ehesten als einen liberalen Aufklärer verstand. So spricht er im Tagebuch von seinen "liberalen Perfektibilitäts-Ideen" und spottet über Metternich und die "Legitimisten" ( $T g b$. 3739). ${ }^{4}$ Seine sarkastische Grabinschrift auf die "gegenwärtig in Gott ruhende Legitimität [...] Beileidbezeugungen werden nur von

Komplex: Walter Weiss, "Biedermeier(Zeit), Vormärz, (Früh)Realismus?" In: Antipodische Aufklärungen. Festschrift für Leslie Bodi. Hrsg. von Walter Veit. Frankfurt/M. 1987. S. 503-517.

Der Name 'literarisches Biedermeier' wird hier nicht primär in dem allgemeinen Sinn einer Epochenbestimmung gebraucht wie in der monumentalen Darstellung von Friedrich Sengle, Biedermeierzeit. 3 Bde. Stuttgart 1971-80, sondern er soll durchaus im spezifischeren Sinn mit seinen geistigen und weltanschaulichen Konnotationen fruchtbar gemacht werden. Er vertritt eine bedeutende Tendenz der Periode zwischen 1815 und 1848.

3. Vgl. besonders Ottokar von Horneks Preis Österreichs in König Ottokars Glück und Ende, V. 1670-1703. Ich zitiere Grillparzers Dramen nach der Verszählung, die in allen relevanten Ausgaben (Historisch-kritische Ausgabe, Hanser-Ausgabe, Ausgabe des Klassiker-Verlages) mit gelegentlichen geringen Abweichungen übereinstimmt. Orthographie und Interpunktion folgen der Ausgabe Franz Grillparzer, Werke. Hrsg. von Helmut Bachmaier. Bd. 2 u. 3. Frankfurt/M. 1986/87.

4. Die Tagebuch-Nummern beziehen sich auf die Zählung in der Historisch-kritischen Gesamtausgabe der Werke Grillparzers: Franz Grillparzer, Sämtliche Werke. Hrsg. von August Sauer. Zweite Abteilung, Bd. 7-12 (=Tagebücher und literarische Skizzenhefte I-VI). Wien/Leipzig (I-II); Wien (III-VI) 1914-1930. Die Zählung wurde in den meisten modernen Ausgaben (auch Auswahlausgaben) übernommen. 
Blödsinnigen angenommen" ( $T g b$. 3749) oder die Satire "Bittschrift der Spitzbuben", in der die Schurken um hohe Protektion bitten und um Beschäftigung in Anstellungen wie denen, wo sie schon immer "berücksichtigt worden sind. Nämlich: die Diplomatie, Zensur und Polizei, das Brieferöffnungsgeschäft, das Personal der Denunzianten und Vertrauten [...]" ${ }^{\text {"5 }}$ sind unmißverständliche Zeugnisse einer rebellischen Gesinnung.

Viele Eintragungen im Tagebuch erscheinen so kühn und fremd, daß sie kaum Stützpunkte im poetischen oder zumindest im dramatischen Werk zu haben scheinen. Ist es wirklich der Autor des Bruderzwist in Habsburg, der das "neue Schlechte immer schon [...] d[em] schlecht[en] Alte[n]" vorzieht (Tgb. 2856), weil es die Verjährungszeit des letztern unterbricht? Oder der im "absichtliche[n] Stehenbleiben der einzelnen oder der moralischen Person ein Verbrechen an dem Geschlechte, ein Vergehen gegen Gott" (Tgb. 1409) sieht? Wie mit sich selbst uneins der Schöpfer Rudolfs II. oder Libussas, der Anwalt einer auf Vertrauen begründeten Herrschaft gewesen sein muß, demonstriert die folgende Notiz: "In manchen Ländern Europas faselt man noch von der Möglichkeit einer patriarchalischen Regierung, einem blind gläubigen Zusammenleben der Staatsbürger, einer unbewuißt zufriedenen Selbstbeschränkung der Ansprüche der einzelnen" ( $T g b$. 3418). Kaum hat sich der fromme Grillparzer-Leser schockiert damit abgefunden, daß diesmal eine solche Vision Faselei ist, da fährt der Schreiber nachdenklich fort: "Die Mọglichkeit läßt sich nicht ableugnen". Auf der gleichen Linie liegt es, wenn er dem Fürsten Metternich mit ebensoviel Bedauern wie Schadenfreude bescheinigt, daß er durch eigene Schuld seine beiden wichtigsten Ziele vereitelt habe, nämlich: "Niederhaltung des Liberalismus und Erhaltung des status quo". 6

Es ist oft bemerkt und nicht selten beklagt worden, daß Grillparzer in den revolutionären Unruhen von 1848 sehr bald eine konservative Haltung einnahm, $\mathrm{da}$ er die Nöte der Vergangenheit erträglicher als die Übergriffe der Gegenwart fand und daß er angesichts der Auflösung der Ordnung sein Heil bzw. das Heil Österreichs im Lager des Marschalls Radetzky suchte.

Die Greuel der letztverflossenen drei Jahre und die Albernheiten der letztverflossnen zwanzig oder dreißig haben in jedem ehrlichen Manne die Uberzeugung hervorgerufen, daß man sich der Regierung anschließen müsse, und zwar nicht nur so, wie es auch in gewöhnlichen Zeiten die Pflicht jedes ehrlichen Mannes ist, sondern auf bestimmtere, ausgesprochenere Art,

notiert er (Tgb. 4046). Aber man sollte solche Stellungnahmen nicht

5. Franz Grillparzer, Sämtliche Werke. Ausgewählte Briefe, Gespräche, Berichte. Hrsg. von Peter Frank und Karl Pömbacher. Bd. 3. München 1964. S. 101.

6. Wie Anm. 5, S. 1033 . 
mißverstehen, als hätte der Autor zur Zeit der Revolution eine plötzliche innere Wandlung durchgemacht. Schon lange vor den revolutionären Bewegungen traute er den "ehrlichen Männern", besonders denen auf dem Katheder, nicht recht über den Weg und lehnte eine unbeschränkte Lehrfreiheit ab. "Jungen widerstandslosen Gemütern destruktives und albernes Zeug in den Kopf zu setzen", heißt nach Grillparzer die Freiheit zu weit treiben. Da empfiehlt er doch lieber Überwachung (Tgb. 3771). Bekanntlich hat der Dichter ja auch die Zensur nur deswegen abgelehnt, weil es keine guten und verantwortlichen Zensoren gebe, - aus praktischen Erwägungen der Durchführbarkeit, nicht aus grundsätzlichen Bedenken gegen die Einschränkung persönlicher Freiheiten. ${ }^{7}$

Durch sein ganzes Wesen geht der Widerspruch zwischen kritischer Auflehnung und gefühlsmäßigem Bewahrenwollen. Er mag sich noch so frei gegenüber religiösen Dogmen fühlen und grollend gegen die "Pfaffenpartei" zu Felde ziehen, - wenn er an die Reformation Martin Luthers denkt, so befindet er, sie sei "übereilt" gewesen und habe, unglücklicherweise,

verhindert, daß das Christentum nach und nach das wurde, was eine Religion erst zum Segen für eine gebildete Zeit macht: eine ehrwürdige Gewohnheit, die man beibehält, weil man nichts Besseres weiß, und ohne in ihre Grundlagen und Beweise näher hineinzugehen. (Tgb. 4066)

Hatte er das "Stehenbleiben als Verbrechen [...] ein Vergehen gegen Gott" deklariert, so kann er bei anderer Gelegenheit ebenso überzeugt verfügen: "Man muß Neuerungen überhaupt nur einführen, wenn sie notwendig oder von wesentlichem Nutzen sind, sonst hat das Bestehende die Vorrechte des Natürlichen." ${ }^{8}$ Richard Alewyn nennt Grillparzer einen "mit der Welt und mit sich selbst zerfallenen Menschen". ${ }^{9}$ Seine liberalen Zeugnisse sind der Tradition der Aufklärung verpflichtet. Seine Gefühle und Instinkte korrespondieren mit dem Geist des Biedermeier oder - nach Nadler ${ }^{10}$ und Alewyn - des Barock. Die

7. Vgl. "Über die Aufhebung der Zensur":

"[...] wäre es nicht ein Glück für die Menschheit und die Literatur, wenn durch eine gute Zensur, jedes unzweifelhaft schlechte, jedes verderbliche Werk vor der Verbreitung schon im Keime zerstört würde.

Es soll also eine Zensur bestehen? - Ja eine gute. Da aber eine gute Zensur nicht möglich ist; eine schlechte aber verderblicher als keine, darum keine; aber nur darum."

Wie Anm. 5, S. 1062.

8. Wie Anm. 5, S. 280. Im Zusammenhang mit einer geplanten Rechtschreibereform geäußert, aber bewußt ins Allgemeine übertragen.

9. Richard Alewyn, "Grillparzer und die Restauration". In: R.A., Probleme und Gestalten. Frankfurt/M. 1974. S. 281-198. Zitat S. 289. 
Lebenszeugnisse sind überwiegend dem rationalen Geist verpflichtet, die Dichtungen wachsen stärker aus dem Gefühls- und Gemütsbereich.

In diesen Zusammenhang von Verändern und Bewahren, von Selbstbestimmung und Selbstbeschränkung gehört auch das Problem des Tuns bzw. Nichttuns, das Grillparzers gesamtes dramatisches Werk durchzieht und den Dichter auch in seinen privaten Aufzeichnungen immer wieder zur Auseinandersetzung reizt. Wenn er über Macbeth reflektiert, so beschäftigt ihn die Frage nach dem unterschiedlichen Verhältnis zu Entschluß und Tat beim Mann und bei der Frau ( $T g b$. 218). In Hamlet sieht er, sehr bezeichnend für seine eigene Dichtung, nicht einen Helden, der durch Kleinmut und Kraftlosigkeit in seinem Tun gehemmt ist, sondern den schwermütigen, "zur Untätigkeit verdammt[en]" ( $T g b$. 1415), "durch gerechte Bedenklichkeiten am Handeln gehindert[en]" (Tgb. 3545) Menschen. Für Napoleon waren seine Taten Reizmittel wie für den Branntweinsäufer der Alkohol. Er mußte "immer handeln, wenn er sich nicht selbst verzehren wollte" (Tgb. 1254).

Auch in seinen Dramen fragt Grillparzer nach den Wurzeln des Tuns, nach den verborgenen Ursprüngen des Handelns im Willen und im Trieb; nach den Motiven, Absichten und Zielen der Täter; nach Kraft und Fähigkeit zur Tat. Aber das Psychologische ist hier nur ein Aspekt des Handelns - und kaum der wichtigste. Hofmannsthal wird zwei Generationen später das Tun als Weg ins Leben preisen und diejenigen, die handeln können, den unfruchtbaren Nichttätern, den Ästheten und Lebensdilettanten gegenüberstellen. Bei Grillparzer ist die Unfähigkeit zum Tun noch nicht zum zentralen Problem avanciert wie im Fin de Siècle. Im Mittelpunkt steht weniger das Tunkönnen als das Tundürfen. Wie wundert der Hispanophile sich doch, daß bei den spanischen Dramatikern die Gewalt - ganz unchristlich - nicht nur geduldet, sondern geradezu gerechtfertigt wird. "Die Lieblingsfiguren ihrer Stücke lassen sich nicht selten entsetzliche Dinge zuschulden kommen, und gegen das Ende unterliegt die Absolution keinem großen Anstande. Besonders hat der Liebende zu allem ein Recht..." (Tgb. 1377). Bei Grillparzer ist das Tun eigentlich immer Frevel, wenn es nicht gerade wie im Fall von Rudolf I. in König Ottokars Glück und Ende in eine historische Idylle eingebunden werden kann. Stets wird der ehrgeizig Handelnde an dem Bewahrenden und Erhaltenden gemessen, der leichtsinnige Empörer dem verantwortungsbewußten Pfleger gegenübergestellt. Eine Problematik des Biedermeier? Ohne Zweifel! Ruhe, zwar nicht unbedingt als erste Bürgerpflicht, aber als harmonischer Naturzustand und Frieden - sowohl als politischer Frieden des Landes wie als innerer Seelenfrieden, als goldene Innerlichkeit - stehen in allen Tatdramen hoch im Kurs. Die Erkenntnis der eigenen Grenzen, die Selbstbescheidung ist Voraussetzung der Wirklichkeitsbewältigung.

Im Jahre 1822, also zehn Jahre vor Goethes Tod, notiert Grillparzer - nicht

10.

Josef Nadler, Franz Grillparzer. Vaduz 1948. 
gerade biedermeierlich bescheiden, aber hundertprozentig aus biedermeierlichem Geist - Ideen zu einer Fortsetzung von Goethes Faust. Faust sollte nach der Gretchentragödie "in sich zurückkehren" und erkennen, "worin eigentlich das Glück besteht: in Selbstbegrenzung und Seelenfrieden." Er sollte

in die Familie eines wackern Hausvaters eintreten [...], wo sich ihm das Glück der häuslichen Liebe kundtut, wo die Tochter, Gretchen ähnlich von Gestalt und einfacher Güte, sich innig an ihn anschließt, anfangs mit kindlicher, dann mit Liebe der Geliebten.

Leider wird aber aus der Idylle nichts, weil Grillparzers Faust im Bewußtsein seiner "Verworfenheit" und seines "Unwerts" die Flucht ergreift und sich selbst in opferbereitem Edelmut paktgemäß dem Teufel ausliefert (Tgb. 1083). Der Tatmensch Faust ist hier zum idyllischen Moralisten degeneriert, und wenn die Erinnerung den Dichter nicht trügt (er spricht davon, daß er sich schon seit etwa zehn Jahren mit dem Plan trägt), dann hat er das Biedermeier als Lebensform in den Stoff eingebracht, bevor das Biedermeier als Epoche (sofern es das gibt) auch nur aus der Taufe gehoben war.

Psychologie und Ethos - Ethos noch mehr als Psychologie - sind die zwei Hauptkomponenten, von denen die Tatproblematik und die Wirklichkeitsproblematik überhaupt bei Grillparzer lebt. Wie diese beiden Aspekte sich in der Frage nach dem Tun durchdringen und ergänzen, soll nun kurz skizziert werden. Wer die Wahl hat, hat bekanntlich die Qual: Man könnte fast alle Dramen zu diesem Unternehmen heranziehen. Ich beginne mit der Ahnfrau nicht in erster Linie, weil sie historisch am Anfang steht, sondern weil in dem Gespensterstück die Frage nach dem Tun eigenwillig und ungewöhnlich gestellt ist. König Ottokars Glück und Ende und Der Traum ein Leben spiegeln einander in den charakteristischen Merkmalen des Tatproblems und sollen zusammen betrachtet werden. Der Bruderzwist in Habsburg ist das reichhaltigste und vielschichtigste Werk für unser Thema und deshalb geeignet, eine Summe zu ziehen.

Der Plot der Ahnfrau beginnt mit einer Gewalttat - und zwar nicht der Titelheldin, sondern ihres Eheherrn, der seine untreue Gattin kurzweg erdolcht. Der Frevel wird freilich ihr angerechnet ob ihrer verbrecherischen Liebesaffäre. Von ihm ist weiter nicht die Rede. Die Geschichte endet wiederum mit einem Gewaltakt. Der letzte Sproß des Hauses, Jaromir, schickt sich an, das Gespenst der Urmutter zu vergewaltigen. Damit schließt sich ein Rahmen, der ein einmaliges Aufgebot an Taten und Verbrechen einfaßt: menschenvernichtendes Räubertum, leidenschaftliche Kampfesszenen, wildeste Drohung, Vatermord und Inzest. In dem ersten vollendeten Drama des Dichters wird nicht lange gefackelt. Der triebhafte Wille scheint völlig ungebändigt. Jaromir ist zweifellos der am wenigsten gehemmte Tatmensch, den man bei Grillparzer antreffen kann. 
Der Autor, der sich selbst gern als Hypochonder darstellt, hat sich heimlich mit dieser Figur identifiziert.

Ich glaube, daß das Genie nichts geben kann, als was es in sich selbst gefunden, und $\mathrm{da} B$ es nie eine Leidenschaft oder Gesinnung schildern wird, als die er selbst, als Mensch, in seinem eigenen Busen trägt [...] Also sollte Shakespeare ein Mörder, Dieb, Lügner, Verräter, Undankbarer, Wahnsinniger gewesen sein, weil er sie so meisterhaft schildert? Ja! Das heißt, er mußte zu dem allen Anlage in sich haben [...] Nur ein Mensch mit ungeheuren Leidenschaften kann meiner Meinung nach dramatischer Dichter sein, ob sie gleich unter dem Zügel der Vernunft stehen müssen und daher im gemeinen Leben nicht zum Vorschein kommen. (Tgb. 221)

Diese Aufzeichnung entstammt dem Jahr 1817, also dem Jahr der Uraufführung der Ahnfrau. Die Begegnung mit dem, wie er meinte, "eigenen lebensgroßen Bild" auf der Bühne verursachte dem Autor eine "unbeschreiblich widerliche Empfindung", und er fand, "es sei ebenso unschicklich, das Innere nackt zu zeigen als das Äußere" (Tgb. 204). Hier leuchtet Heinz Politzers Interesse an dem "abgründigen Biedermeier" bei Grillparzer unmittelbar ein. ${ }^{11}$

Man hat Jaromir bisweilen als "edlen Räuber" gedeutet, der wie Schillers Karl Moor aus Verzweiflung zum Verbrecher wird und gegen eine ungerechte Welt zu Felde zieht. Diese Interpretation läßt sich durch den vorteilhaften Eindruck täuschen, den der Held anfangs auf Berta und Borotin macht, und durch seine unschuldige Entführung als Kind, die Tatsache, daß er zugleich ein Edelmann und ein Räuber ist - vielleicht auch durch seine eigenen sentimentalen Worte, die den Räuber als "Stiefsohn des Geschicks" bedauern (V. 1325). Aber alle Berichte über Jaromirs mörderische Vernichtungszüge widersprechen dem. Sein Tun ist triebhaft. In keinem Augenblick widersteht er aus Rücksichten auf seine Mitmenschen einem Gewaltakt. Weder die geliebte - oder wenigstens heiß begehrte - Berta noch Borotin haben einen Einfluß auf ihn, wenn er seinen Wünschen nachjagt, wenn er in den Sog seines Tuns gerät.

Das einzige Talent, das in ihm ebenso stark entwickelt ist wie sein blinder Tatendrang, ist die Macht des Wortes. Die Worte stellen sich ihm aber nicht hindernd vor die Taten wie später den Figuren Hofmannsthals, sondern sie steigern seinen Tatendrang: er redet und begeistert sich in seine Handlungen hinein. Zugleich nutzt er seine hinreißenden sophistisch-rhetorischen Fähigkeiten, um Berta zu überreden, zu überzeugen und zu erpressen. Seine Rhetorik wächst wie sein Tun zwanghaft aus dem triebhaften Wollen, steht aber immer im Dienst seines zweckhaften Kalküls. Nicht weniger als das Tun selbst übt sie Zwang aus.

Wir dürfen Jaromir nicht beim Wort nehmen; denn die Widersprüche seiner Reden sind Legion. Wenn er Bertas Mitleid gewinnen will, indem er sein

11. Heinz Politzer, Franz Grillparzer oder das abgründige Biedermeier. Wien 1972. 
Räubertum mit dem Vorbild derer, "die er liebte" (V. 1905) entschuldigt, andererseits seinem räuberischen Pflegevater versichert, daß er ihn schon immer gehaßt habe, - wenn er als Räuber innerlich gerungen und gelitten haben will, dann aber seiner Geliebten damit droht, daß ein blutdürstiger Löwe nur ein Schoßhund sei gegen ihn ("Und der Nächste meinem Herzen / Ist der Nächste meinem Dolch", V. 3239-40), so erkennen wir, daß seine Worte aus der jeweiligen Situation hervorgehen und dem jeweiligen Zweck dienen.

Jaromir ist der einzige Tatmensch bei Grillparzer, der nie die Verantwortung für sein Tun übernimmt und auch vom Dichter nicht zur Verantwortung gezogen wird. Von dem Vatermord will er nichts wissen: "Ha, getan! - Hab ich's getan? / Kann die Tat die Schuld beweisen, / Muß der Täter Mörder sein?" fragt er (V. 2976-78) und kommt nach beträchtlichem rhetorischen Aufwand zu dem Schluß:

$\mathrm{Ja}$, der Wille ist der meine,

Doch die Tat ist dem Geschick [...]

Unsre Taten sind nur Würfe

In des Zufalls blinde Nacht.

$\mathrm{Ob}$ sie frommen, ob sie töten?

Wer weiß das in seinem Schlaf!

Meinen Wurf will ich vertreten,

Aber das nicht was er traf!

Dunkle Macht, und du kannst's wagen,

Rufst mir Vatermörder zu?

Ich schlug den, der mich geschlagen,

Meinen Vater schlugest du! (V. 2997-3012)

Diese Reflexionen, die deutlich auf Hofmannsthals Griechendramen eingewirkt haben, klingen zunächst wie die typische, den eigenen Vorteil suchende Sophistik Jaromirs, die wir schon kennen. Es steht dem Helden, der wenig später bereit ist, seine Schwester zu verführen und die Ahnfrau zu vergewaltigen, nicht an, sich auf seine Unschuld zu berufen. Dennoch steht er diesmal nicht allein. Der Ermordete, der alte Borotin, kommt sterbend zu demselben Ergebnis: "Seht ihr mich verwundert an? / Das hat nicht mein Sohn getan! / Tiefverhüllte, finstre Mächte / Lenkten seine schwanke Rechte!" (V. 2540-43) - Die Schicksalszusammenhänge in Grillparzers Trauerspiel sind nicht schlüssig (ich kann auf diesen Komplex hier nicht näher eingehen ${ }^{12}$ ), aber unbestreitbar sind Kräfte am Werk, die der einzelne nicht kontrollieren kann. Die Erscheinung des Gespenstes im Schloß bedeutet, wie es heißt, stets "Unglück oder Freveltat" (V.

12. Vgl. meinen Aufsatz: "Grillparzers Ahnfrau. Ein fatalistisches Schauerdrama." In: Zeitschrift für deutsche Philologie 107 (1988), Sonderheft. S. 45-61. Darin weitere Literaturangaben zur Schicksalsproblematik. 
2180); Unglück entzieht sich der individuellen Verantwortung; einen Frevel hat der Täter zu verantworten. Beide durchdringen einander hier; die Tat wird, mit Fülleborns Begriffen, zum "Geschehen". Grillparzers Ahnfrau ist wohl das einzige Werk des Dichters, in dem die Ambivalenz des Getanen und Geschehenen unaufgelöst bleibt.

Als Gegenwelt zu dem Bereich von Gewalt und Verbrechen wird immer wieder die friedliche Idylle beschworen, und zwar von niemandem häufiger als von dem grausamen Jaromir. Woher der Räubersprößling eigentlich seine kitschigen Vorstellungen von der "Menschheit süßen Pflichten" (V. 1897), von "der Sitte heil'gem Brauch" (V. 1899) hat, wie der Pflegesohn Boleslavs zu seinem Hymnus auf das Vatertum kommt, das fragt sich der Leser oder Zuschauer vergebens:

\author{
Was die Erde Schönes kennet, \\ Was sie hold und lieblich nennet, \\ Was sie hoch und heilig glaubt, \\ Reicht nicht an des Vaters Haupt. \\ Balsam strömt von seinen Lippen \\ Und auf wem sein Segen ruht, \\ Der schifft durch des Lebens Klippen \\ Lächelnd ob der Stürme Wut. (V. 2942-48)
}

Wahrscheinlich hat Jaromir in der Einsamkeit der Wälder viel Schiller gelesen. Es gibt manche falschen Töne in diesem Konzert der Stimmen. Der betende und moralisierende Räuber, der manchmal dieselben Normen hat wie alle frommen Gemüter und um nichts mehr besorgt ist als um seiner "Seele goldnen Frieden" (V. 2841), sich manchmal aber seelenlos wie ein reißendes Tier aufführt, ist psychologisch nicht glaubwürdig. Aber der Wirkung des Dramas tut dieser Widerspruch keinen Abbruch.

Wir dürfen nicht vergessen, daß es sich bei der Ahnfrau um ein fatalistisches Schauerdrama handelt. Was in einem (im engeren Sinn) psychologischen oder Weltanschauungsdrama verderblich wäre, erscheint in diesem Genre eher gerechtfertigt. Grillparzer sucht den Effekt, die Atmosphäre, die Wirkung auf Sinne und Nerven. Er gebraucht alle atmosphärischen Mittel des Schauerlichen und Gespenstischen, die ihm zur Verfügung stehen. Werner und Müllner sind, so sehr der Dichter sich auch dagegen sträubt, diese Verbindung zuzugeben, Vorbilder seines Schaffens. ${ }^{13}$ Als Kontrast zu dem Unheimlichen und Grellen braucht er die friedlichen, idyllischen Töne, so daß die Erregung immer neu stimuliert werden kann. Der tragische Widerspruch zwischen dem edlen Retter, den Berta und Borotin zunächst in Jaromir sehen, und dem erbarmungslosen Gewaltmenschen, als der er sich entpuppt, trägt zu dieser grausigen Spannung des Stückes bei. Und die

13. Wie Anm. 12. 
Ambivalenz des Tuns zwischen Freveltat und Laune des Geschicks fügt sich wirkungsvoll in das Konzept des Ganzen.

In König Ottokars Glück und Ende und Der Traum ein Leben ist von Ambivalenz wenig zu spüren. Wie im volkstümlichen Märchen können wir uns jederzeit leicht orientieren. Wir wissen, wer recht handelt und wer unrecht hat, und wir können die Motive der Figuren klar durchschauen. Der Traum ein Leben heißt, obwohl es sich bei dem Werk ja ganz konkret um ein Traumgeschehen handelt, "dramatisches Märchen". Der Untertitel verbindet das Drama mit dem Wiener Volkstheater. Von dem historischen "Trauerspiel" König Ottokars Glück und Ende sagt Politzer überzeugend: "All das ist kein historisches, sondern ein in seiner theaterkundigen Naivität höchst raffiniert gebautes Heiligen- und Märchenspiel." 14 Wie berechtigt der Name "Trauerspiel" eigentlich ist, bleibt dahingestellt. Die Böhmen mochten trauern, die Österreicher konnten sich freuen. Jedenfalls nähern die beiden Dramen sich genre- und strukturmäßig an, und man darf auch mehr als einen Zufall darin sehen, daß beide einen barock eingefärbten Titel tragen. Die Variation von Calderóns Das Leben ist ein Traum ruft die barocke Leben-Traum-Gleichung ins Gedächtnis, und König Ottokars Glück und Ende verweist auf die barocke Idee der Vanitas, der Unbeständigkeit des irdischen Glücks. Wie dieses Barock sich schließlich doch zum Biedermeier umbildet, wird später zur Sprache kommen.

Beleuchten wir zunächst die Verwandschaft noch etwas näher: Die beiden Hauptfiguren sind ehrgeizige Menschen, die sich mit dem, was sie sind und was sie besitzen, nicht zufrieden geben. Wie Rustan, der Sohn und Enkel von Kriegern, sich aus der ländlichen Ruhe seines Oheims in die Welt des Krieges und des Ruhms hinaussehnt, so trachtet Ottokar über seine böhmische Kriegswürde hinaus ins 'Karlische', 'Karl-der-Großische', d.h. ins Grenzen- und Maßlose. Beide wollen "wie ein Gott" ins Leben greifen. Zeichenhaft für ihren politischen Irrweg erscheint im privaten Bereich jeweils die Lösung von einer treuen weiblichen Seele und die Verbindung mit einer scheinbar viel begehrenswerteren Frau, die ihnen aber verderblich wird. Rustans Abwendung von Mirza und seine Faszination durch Gülnare spiegeln durchaus Ottokars Weg von Margarethe zu Kunigunde. Der wichtigste Unterschied der Werke liegt in ihrem Ende. Im märchenhaften Traumstück darf der Held sich bessern, um in Gnaden wieder aufgenommen zu werden in den Kreis der edleren Menschen. In der historischen Welt lassen sich Taten nicht rückgängig machen. Ottokar wird zwar auch mit Erkenntnis gesegnet und zeigt Reue, aber untergehen muß er nichtsdestoweniger.

Peter von Matt ${ }^{15}$ Gerhart Baumann ${ }^{16}$ und Fülleborn haben den Rhythmus

14. Heinz Politzer, Franz Grillparzer oder das abgründige Biedermeier. Wien 1972. S. 168.

15. Peter von Matt, Der Grundriß von Grillparzers Bühnenkunst (=Zürcher Beiträge zur deutschen Literatur- und Geistesgeschichte, hrsg. von Emil Staiger, Nr. 24). 
einer typischen Grillparzer-Handlung beschrieben: Die weltlichen Helden des Dichters lösen sich aus den Ordnungen ihres Daseins, blicken mit Verachtung auf ihre Vergangenheit zurück, "ohne indes fähig zu werden, der neuen Göttin zu opfern." 17 So geraten sie fortschreitend in Verwirrung, Lüge, Reue oder Heimweh. Baumann spricht in Anlehnung an Ferdinand Kürnberger von einer "Revolution auf der Umkehr." ${ }^{18}$ In bezug auf dieses Modell gibt es wohl keine typischeren Grillparzer-Dramen als die hier diskutierten. In beiden machen die Hauptfiguren eine Fehlentwicklung durch. Rustan war einst, wie wir von Mirza hören, "sanft [...] fromm und mild" (V. 109), und Ottokar galt dem jungen Seyfried als Muster und Vorbild "von jedem hohen Tun" (V. 21). Aber die Aussicht, mehr zu haben und mehr zu sein, verdirbt ihre Tugend. Gleich der erste Schritt auf dem Weg zur Größe erweist sich als Vergehen gegen menschliches und göttliches Recht, das eine Kette von unheilvollen Konsequenzen nach sich zieht. Sie handeln nicht blind. Aber die Lockung ist so groß, daß sie gegen ihr besseres Selbst agieren. Rustan weiß, daß ein anderer den Fürsten von Samarkand gerettet hat, doch in der Hoffnung auf die Dankbarkeit des Königs und die Liebe Gülnares annektiert er die Tat und ermordet den wirklichen Retter. Ottokar ist sich bewußt, daß die vorgegebenen Gründe für die Verstoßung Margarethes nur Fiktion sind, doch er maßt sich an, sich über Gesetz und Moral hinwegsetzen zu dürfen.

Doch nun offenbart sich etwas Eigentümliches. Die Ungerechtigkeit der Tat, das Tatverbot nimmt dem Tun zugleich alle Kraft. Der ungerechte Täter ist in beiden Dramen, wie fast immer bei Grillparzer, ein schwacher Täter. Bei Rustan war das wohl vorauszusehen. Seine Ängstlichkeit gegenüber dem Zorn des Oheims, sein eitles Schwärmen, seine Offenheit für die Schmeichelei des Ohrenbläsers Zanga ließen keine großen Heldentaten erwarten. Es überrascht kaum, daß er auf jeder Stufe seines Weges in die Welt versagt und sich nur durch Betrug eine Zeitlang halten kann. Grillparzer entlarvt in ihm den politischen Tatmenschen als verantwortungslosen Frevler und Ränkeschmied, der selbst nicht mehr Herr seines Tuns ist. Ottokar aber schien ein kraftvoller Herrscher, solange er sich nicht überhob. Erst sein Übermut, seine Verachtung des Rechts scheinen ihn schwach zu machen. Darin liegt eine Lehre und direkte Warnung des Autors! Die Szene der großen Machtentfaltung und der willkürlichen Selbsterhebung markiert auch hierin den Wendepunkt. Von jetzt an zeigt Ottokar keinerlei Eigeninitiative mehr, sondern reagiert nur auf das, was seine Gegenspieler tun.

\section{Zürich 1965.}

16. Gerhart Baumann, Franz Grillparzer: Dichtung und österreichische Geistesverfassung. Frankfurt/M. 1966. (=Uberarbeitete und erweiterte Fassung des Werks von 1954)

17.

Peter von Matt, S. 125.

18. Gerhart Baumann, S. 101. 
Und er reagiert unbegreiflich schwach. Seine Blindheit oder seine Nachsicht gegen Zawisch und Kunigunde, die tatenlose Hinnahme jeder Kränkung, sein Wunsch, die hartherzige Gattin solle ihn süß in Schlummer plaudern, grenzen ans Komische. Die Auseinandersetzung mit dem neuen Kaiser erschöpft sich in einer müden Aufwallung seines Zorns. Er demütigt sich und empört sich zugleich gegen die Demütigung - beides halbherzig und ohne Konzequenz. Die endliche Einkehr und Reue nähern sich dem Unglaubwürdig-Sentimentalen.

Können solche schwächlichen Tatmenschen wirklich den politischen Ehrgeiz und das Tun diskreditieren? Zumindest sind sie dazu konzipiert! Die vielzitierten Verse des aus gefährliche Träumen erwachten Rustans haben manchen Interpreten verärgert und zu scharfsinnigen Widerlegungsversuchen angefeuert. ${ }^{19}$ Dennoch enthalten sie zweifellos die Lehre des Stücks:

\author{
Eines nur ist Glück hienieden, \\ Eins, des Innern stiller Frieden, \\ Und die schuldbefreite Brust. \\ Und die Größe ist gefährlich, \\ Und der Ruhm ein leeres Spiel; \\ Was er gibt, sind nichtge Schatten, \\ Was er nimmt, es ist so viel. (V. 2650-56)
}

Wenn Grillparzer diese Lektion dem Lebensweg eines kraftlosen Wunschtäters abgewinnt, so wird sie dadurch nicht relativiert, sondern gewinnt im Gegenteil eher an allgemeingültiger Relevanz. Und ihre Gültigkeit wird auch kaum durch die oft geäußerten Zweifel beeinträchtigt, ob ein Mensch wie Rustan wirklich nach diesen Prinzipien wird leben können. Rustans Worte entsprechen jedenfalls genau der Vanitas-Botschaft des Derwischs, die am Anfang des Stückes den weltanschaulichen Horizont ausmißt:

Schatten sind des Lebens Güter,
Schatten seiner Freuden Schar,
Schatten Worte, Wünsche, Taten;
Die Gedanken nur sind wahr. (V. 628-31)

Diese Entwertung des irdischen Strebens wird meist als barocke Lehre gedeutet. Aber hören wir genau auf den Wortlaut: Als Alternative der schattenhaften Freuden, Worte, Wünsche, Taten wird nicht wie im Barock die göttliche Welt, nicht die transzendente Wahrheit beschworen, sondern das Reich der Gedanken. Wir haben hier ein säkularisertes Barock vor uns, d.h. aber zugleich

19. Vgl. besonders Reinhard Urbach, "Zufriedenheit bei Ferdinand von Raimund ". In: Austriaca. Beiträge zur österreichischen Literatur. Festschrift für Heinz Politzer. Hrsg. von Winfried Kudzus u. Hinrich Seeba. Tübingen 1975. S. 107-126. 
echtes Biedermeier. Denn im Biedermeier leben die Werte und Beurteilungen des Barocks weiter, während sich zugleich der religiöse Horizont mehr oder weniger aus den Augen verliert. Die Moral bedarf nicht mehr selbstverständlich der Rechtfertigung aus der göttlichen Ordnung, sondern sie gründet im Irdischen oder eigentlich in sich selbst. Im biedermeierlichen Volkstheater können deswegen aus den göttlichen und teuflischen Mächten der barocken Vorstellungswelt gute und böse Geister werden.

Peter von Matt behauptet: "Die eigentlichen Helden Grillparzers sind [...] nicht die Ehrfürchtigen, sondern die Vermessenen, die in tragischem Wahn von Freiheit befangenen Täter." ${ }^{20}$ Andere Forscher scheinen davon weniger überzeugt. Doch auf jeden Fall lohnt es sich, einen kurzen Blick auf die friedlicheren Gegenfiguren zu den gewaltsamen Tatmenschen zu werfen. - Die Welt von Massud und Mirza ist der ländliche Bereich von Haus und Feld. Die Menschen leben in Einklang mit einer idyllisch verklärten Natur still, ruhig, zufrieden dahin. Die Landarbeit mag mühsam sein, aber die Anstrengungen werden durch inneren Frieden belohnt. Man ist wohlhabend, aber bleibt bescheiden. Man fühlt sich als Familie verbunden, und Milde, Sanftheit, Güte und Rücksicht bestimmen das Zusammenleben. Es ist aus moderner Sicht Rustan nicht gerade uibelzunehmen, daß er nicht immer "still und ruhig" (V. 141) sitzen und sein Leben wunschlos in diesem engen Kreis beschließen möchte. So mancher andere würde sich in diesen Verhältnissen gerade so langweilen wie er. Alle Tätigkeit, zu der die biedermeierliche Idylle Raum läßt, ist das "stille Tun und Treiben" (V. 467) des Landwirts und Familienvaters, das auf den Besitz eines ruhigen Glücks zielt. Hier steht Grillparzer dem Dichter des sanften Gesetzes ganz nahe.

In König Ottokars Glück und Ende wird nun - ein erstaunliches Unterfangen - dieses Ideal auf die politische Welt und den höchsten Würdenträger des Reiches, Kaiser Rudolf I., projiziert. Grillparzer hat den ersten Habsburger König (Kaiser ist er nie gewesen) aus einem klugen und energischen Machtpolitiker zum biedermeierlichen Landesvater verwandelt. Rudolf, der zum höchsten weltlichen Rang aufgestiegen ist, erhält sich persönliche Demut und Bescheidenheit. Wie einen einfachen Soldaten läßt der Autor inn im Zelt sitzen und mit dem Hammer seinen zerbeulten Helm ausklopfen. Und sein Amt vergleicht er mit dem eines verantwortungsbewußten Landwirts, der für schlechte Zeiten vorsorgt. Das oberste Ziel des Kaisers ist, "Ruh und Recht" zu schirmen (V. 1809); denn der Begriff des Rechts wird in diesem Drama noch nicht wie später aus der Unvollkommenheit abgeleitet; das Recht ist uneingeschränkter Wert. Weil der Kaiser gegenüber Ottokar das moralisch bessere Prinzip vertritt, braucht er gar nicht viel zu tun, um seinen Gegner zu überwinden. Das Unrecht zerstört sich - eine optimistische Vision - geradezu von selbst. Der Kriegerische unterliegt dem Friedliebenden, der

20.

Peter von Matt, S. 125. 
Ehrgeizige dem Genügsamen. Dennoch darf man den Kaiser zu den Tätern zählen. Zwar weiß er - und das hat er vor dem verblendeten Ottokar voraus -, daß "der Helden, der Gewaltgen Zeit dahin" ist (V. 1916), daß die "neue Zeit" (V. 1920) auf bäuerliche und bürgerliche Tätigkeit, auf Handel und Gewerbe gestellt ist, aber er ist ein Beweis dafür, daß energisches Handeln und Ethos sich bei Grillparzer nicht ausschließen müssen. Es gibt die Verbindung von Biedermeier und Tat. Das selbstlose Tun aus der Verantwortung für die Menschheit harmoniert uneingeschränkt mit dem biedermeierlichen Bedürfnis des Bewahrens, des Erhaltens und der Beförderung des Gesamtwohls. ${ }^{21}$

Rudolf I. hat - und das ist interessant - einen Nachkommen, einen nahen Verwandten in Grillparzers Werk. Wie der Kaiser gebraucht dieser uneigennützig seine Tatkraft zum Wohl des Volkes; wie jener vereinigt er den Landmann und den Fürsten in einer Person, und wie Rudolf sucht er den Begriff des Rechts zu neuen Ehren zu bringen. Es ist Primislaus in dem Libussa-Drama. In einem anderen Zusammenhang könnte er ein Idealherrscher sein, und nicht selten ist er als solcher mißverstanden worden. Aber in der Konfiguration mit Libussa und ihren weisen bzw. besserwisserischen Schwestern können sich seine Prinzipien nicht entfalten. Grillparzer ist mißtrauisch gegenüber dem Recht geworden und gegenüber allem Praktischen und Nützlichen. Deshalb bleibt dem Primislaus bei aller geistigen Nähe zu Kaiser Rudolf nicht nur der Glanz versagt, der jenen umleuchtete, sondern seine Tätigkeit wird überhaupt nur widerwillig und mit Vorbehalten gewürdigt.

Das Vertrauen in die Mächte der Geschichte, das aus König Ottokars Glück und Ende sprach, ist in Grillparzers zweitem historischen Drama einem tiefen Pessimismus gewichen. Der Titel Ein Bruderzwist in Habsburg mag auf eine vorübergehende Krise hindeuten, und die visionäre Habsburg-Prophezeihung "Mein Haus wird bleiben, immerdar" (V. 1277) klingt zuversichtlich, aber diese Voraussage hat keine Basis in dem Drama und bildet schwerlich ein Gegengewicht zu der negativen Entwicklung.

Seit Hofmannsthals Grillparzer-Rede aus dem Jahre 1922 betrachtet man den alten Dichter meist als den eigentlichen Grillparzer. Mit keinem Werk außer vielleicht dem Armen Spielmann hat man ihn häufiger identifiziert als mit dem Bruderzwist. Die Gestalt Rudolfs II., die Fülle seiner Gedanken und Gefühle, sein Grübeln und seine Menschenscheu haben so viele Entsprechungen in Grillparzers eigener Persönlichkeit, daß man versucht ist, die eine für die andere gelten zu lassen. In diesem komplexesten unter Grillparzers Dramen ist auch die Problematik des Tuns und des Nichttuns am weitesten entfaltet und am differenziertesten gestaltet. Persönliche und überpersönliche Voraussetzungen der Tat, die ethischen Prinzipien, die Folgen, die sozialen und die religiösen

21. Auch hier leuchtet die Parallele zu Stifter unmittelbar ein. Man vergleiche die uneigennützige Tätigkeit und die Tatkraft der Gutsbesitzer in Stifters Brigitta. 
Zusammenhänge des Handelns bieten ein reiches Erkenntnisfeld. Da jedoch dieses Drama, seiner Bedeutung entsprechend, vielfach interpretiert ist, kann ich mich mit gutem Gewissen kurz fassen.

Das allgemeine Thema des Bruderzwists ist der Einbruch einer neuen Zeit, aber nicht wie in König Ottokars Glück und Ende einer Zeit des Aufbaus, der Ordnung, der Rückbesinnung auf das Alte, Gute, sondern einer Zeit der Willkür, der Eigensucht, des Kriegs und des Chaos. Das Neue ist zugleich das Schlechte. Kaiser Rudolf II. steht allein für die vergehende Ordnung, sucht zu retten und zu bewahren, aber fühlt sich gelähmt von Furcht vor dem anstürmenden Grauenvollen und von dem Mißtrauen in seine eigenen Kräfte. Die neue Zeit hat viele Dimensionen und viele Helfer. Alle Personen stehen in ihrem Sold. Matthias vertritt die politische Verschlechterung. Getrieben von Eitelkeit und Tatendurst ohne Tatkraft und Verantwortungsgefühl, entstammt er unverkennbar dem Geschlecht Rustans und Ottokars. Don Cäsar, der natürliche Sohn des Kaisers, der sich mit allem, was schlecht oder verboten ist, verbündet, repräsentiert das moralische Gegenspiel. Und da Grillparzers Weltanschauung im Moralischen ihr Zentrum hat, kann Don Cäsar gelegentlich die neue Zeit selbst verkörpern. Ferdinand, der fanatische Eiferer für den überlieferten Glauben, stellt die religiöse Intoleranz und das Inhumane der Epoche dar. Den Protestanten wird die religiöse Spaltung angekreidet, und die Stände mit ihren Rechtsforderungen haben die soziale Fehlentwicklung zu verantworten - bis hin zur Revolution des Proletariats. Im Spiegel des frühen 17. Jahrhunderts setzt sich Grillparzer, wie ja bekannt, mit den sozialen und moralischen Problemen seiner eigenen Zeit auseinander, und er bekennt sich zu einem konservativen Ideal, zum Bewahren des status quo, zu einer statischen Welt. Da die neue Zeit einerseits böse, andererseits übermächtig ist, muß sie bekämpft, kann aber nicht besiegt werden. So wird sie von Rudolf 'in effigie', nämlich in der Person Don Cäsars hingerichtet. Im Einzelfall kann der passiv zögernde Kaiser zum Entschluß und zur Tat schreiten, dem Ganzen gegenüber bleibt er ohnmächtig.

An Anfang des Dramas ist Rudolf in seinem Kaisertum unangefochten, und die verschiedenen Mächte, die feindlichen Parteien im Innern ebenso wie die Armeen im Türkenkrieg, befinden sich im Gleichgewicht miteinander. Es geht herzlich schlecht, aber es geht zumindest. Am Ende steht es um das Reich wesentlich schlimmer. Wenn sich die Situation verändert und verschlechtert, so liegt das in erster Linie an denen, die sich berufen fühlen, einzugreifen und zu handeln, an den Tatmenschen. Der ebenso schwärmerische wie schwächliche Matthias, schwankend zwischen Enthusiasmus und Resignation, wird mit Hilfe seines Beraters Klesel zum Verräter am Kaiser und stürzt das Land in den Bürgerkrieg. Der eindimensional denkende und deshalb entschlußfreudige Ferdinand glaubt wegen der Passivität Rudolfs in eigener Regie handeln zu dürfen und greift 
brutal in die religiösen und politischen Auseinandersetzungen. Leopold, sein loyaler Bruder, möchte dem Kaiser helfen und verschlimmert durch sein Eingreifen nur die Situation. Alles politische Handeln in dem Drama ist moralisch problematisch und wirkt sich negativ für das Ganze aus, trägt zur Zerstörung bei. Auf diesem Hintergrund muß man Rudolfs Worte lesen:

Ich bin so gut nicht als es etwa scheint -

Die Andern nennens schwach, ich nenn' es gut.

Denn was Entschlossenheit den Männern heißt des Staats

[und die folgende Beschreibung zielt genau auf Matthias, Ferdinand und Leopold]

ist meisten Falls Gewissenlosigkeit [Matthias],

Hochmut [Ferdinand] und Leichtsinn [Leopold], der allein nur sich

Und nicht das Schicksal hat im Aug der Andern;

Indes der gute Mann auf hoher Stelle

Erzittert vor den Folgen seiner Tat. (V. 1695-1702)

Tat ist Hybris, die aus einer Überschätzung des Ichs hervorgeht. Als einseitige Überzeugung tendiert dieses Ichgefühl zu bedenkenlosen Entschlüssen, als triebhaftes Wollen zur raschen, unbedenklichen Aktion. Eins ist so problematisch wie das andere. Rudolf ist selbst nicht frei von der Versuchung, den Impulsen seines Ichs zu folgen. Aus Verärgerung über den Verrat des Matthias, aus Enttäuschung und Groll über den Eigennutz der Stände läßt er sich von Leopold die Erlaubnis abringen, mit seinem Heer zu Hilfe zu kommen, und trägt damit zu dem Kriegsübel bei, das er gerade vermeiden wollte. Hier handelt er gegen seine Erkenntnis, die ihm sonst so teuer ist. Rudolf mag sich als "schwacher, unbegabter Mann" (V. 351) fühlen, dem eine zu große Aufgabe auferlegt ist. Zweifellos ist sein Rückzug aus den Regierungsgeschäften, seine Beschäftigung mit privaten Liebhabereien eine sehr unkaiserliche Resignation. Aber als tiefste Wurzel seiner Passivität sollen wir doch seine Erkenntnis, seine Weisheit verstehen. Nicht aus Gleichgültigkeit, sondern aus der Überzeugung heraus, daß jede Bewegung, jede Tat das prekäre politische und soziale Gleichgewicht erschüttern würde, hält er sich still. Er erinnert darin an Hamlet, wie Grillparzer ihn versteht, der durch gerechte Bedenken am Tun gehindert wird. Wo jede Veränderung eine Verschlechterung bedeutet, da gibt es ein Nichttun aus Verantwortung. Da genügt es nach Grillparzer, das Alte zu leben und zu repräsentieren.

Dennoch ist der Gegensatz von Erkennen und Handeln natürlich eine Fiktion. Die Weisheit bleibt, wenn sie sich nicht mit Tätigkeit verbindet, unwirksam. Und so sehr Rudolf offensichtlich aus dem Herzen oder dem Instinkt Grillparzers 
spricht, der Autor durchschaut natürlich die Schwäche seiner Position. Aus Sorge um die Zukunft übersieht Rudolf das Nächstliegende; er schätzt vielleicht das Ganze richtig ein, aber nicht das Aktuelle und Besondere; er hält sich still, doch ermutigt damit gerade die anderen, die schlechter sind als er, zum Handeln. So entsteht ein "Zerrbild" (V. 2293) dessen, was er gewollt hat. In einer von Eigennutz und Rechthaberei zerrissenen Welt mag die Weisheit die höchste Würde behaupten, eine Chance hat sie nicht.

Vermöchte sich irgend jemand in der verworrenen Welt zu behaupten und die Geschicke des Ganzen zu lenken? Einen gibt's, der dazu in der Lage wäre, der Klugheit und Tatkraft verbindet, der die Kunst der Rede ebenso beherrscht wie die politische Aktion, der alles vereinigt, was sich sonst auszuschließen scheint. Es ist der Bischof Klesel, von dem die wichtigsten Unternehmungen in dem Drama inspiriert sind, der seinem Herm Matthias die Kraft einflößt, die seinem Begehren fehlt. Niemand außer Matthias steht ihm nahe, jeder spricht übel von ihm, und auch der Dichter bringt ihm weniger Wohlwollen entgegen als selbst den problematischen Erzherzögen.

Klesel ist unter den Charakteren des Dramas der eigentliche Gegenspieler Rudolfs II. Der Kaiser lebt und denkt als metaphysischer Mensch, dessen Wirklichkeitsauffassung aus der religiösen Betrachtung der göttlichen Ordnung abgeleitet ist. Das irdische Dasein ist für ihn Spiegel des Kosmos. Seine Werte: Frömmigkeit, Demut und Ehrfurcht sind am Sternenhimmel gewonnen. Jede Selbsterhöhung bedeutet Abfall von Gottes Schöpfung. Das Kaisertum ist eine von Gott auferlegte Pflicht. Idealerweise sollten die Menschen, ohne zu rechten, ohne zu prüfen, ebenso selbstverständlich kreisen, leben und verehren wie die "Lämmerherde" (V. 408) der Sterne. Klesel dagegen ist der prinzipienlose Politiker und Pragmatiker katexochen, eine Person, an der ein Grillparzer keine Freude haben kann. Er mag noch so sehr das politisch Richtige und Notwendige erkennen und tun, Verträge schließen und erfüllen, den religiösen Frieden befördem, letzlich bleibt er für alle ein Intrigant. Einem Menschen, dem das sogenannte 'Höhere' abgeht, helfen bei Grillparzer alle tüchtigen Eigenschaften nicht. Das Beste, was er für ihn tun kann, ist, ihn mit einiger Würde abgehen zu lassen. Den biedermeierlich frommen Kaiser dagegen verklärt er am Ende in einer Gloriole, die alle früheren Schwächen vergessen läßt. 